\title{
Murdochiella asaccharolytica gen. nov., sp. nov., a Gram-stain-positive, anaerobic coccus isolated from human wound specimens
}

Correspondence

Paula H. Summanen carlsonph@aol.com

\author{
Nurver Ulger-Toprak, ${ }^{1,2}$ Chengxu Liu, ${ }^{1}$ Paula H. Summanen ${ }^{1}$ \\ and Sydney M. Finegold ${ }^{3,4}$
}

\author{
${ }^{1}$ Research Service, VA Medical Center West Los Angeles, Los Angeles, CA 90073, USA \\ ${ }^{2}$ Marmara University, Faculty of Medicine, Department of Microbiology, Istanbul, Turkey \\ ${ }^{3}$ Infectious Diseases Section, VA Medical Center West Los Angeles, Los Angeles, CA 90073, USA \\ ${ }^{4}$ Departments of Medicine and of Microbiology, Immunology, and Molecular Genetics, UCLA School \\ of Medicine, Los Angeles, CA, USA
}

Gram-positive anaerobic cocci (GPAC) are part of the commensal flora of humans and animals and are also commonly associated with a variety of human infections. They account for about $25-30 \%$ of all anaerobic isolates recovered from clinical samples (Finegold, 1977). Extensive taxonomic changes have occurred recently among this group of bacteria with the addition of novel species and renaming of existing species (Finegold et al., 2002; Murdoch, 1998). Peptostreptococcus productus was transferred to the genus Ruminococcus and the genus Peptostreptococcus was divided into new groups. Currently, five genera of GPAC that contain former members of the genus Peptostreptococcus that may be isolated from humans have been designated: Parvimonas, Finegoldia, Peptoniphilus, Anaerococcus and Gallicola (Ezaki et al., 2001). The only species remaining in the genus Peptostreptococcus are Peptostreptococcus anaerobius and a novel species, Peptostreptococcus stomatis, isolated recently from the human oral cavity (Downes \& Wade, 2006). Three of the new genera, Parvimonas, Finegoldia and Gallicola, have only one species each, Parvimonas micra, Finegoldia magna and Gallicola barnesae,

\footnotetext{
Abbreviations: GPAC, Gram-positive anaerobic cocci; SPS, sodium polyanethol sulfonate.

The GenBank/EMBL/DDBJ accession number for the $16 \mathrm{~S}$ rRNA gene sequence of strain WAL $1855 C^{\top}$ is EU483153.
}

respectively. The type species of the two other genera are Peptoniphilus asaccharolyticus and Anaerococcus prevotii.

We have isolated two GPAC strains, WAL 2038E and WAL $1855 \mathrm{C}^{\mathrm{T}}$, from a human abdominal wall abscess and a sacral pilonidal cyst aspirate. Comparative 16S rRNA gene sequencing studies demonstrated that the unknown strains were genotypically homogeneous but did not appear to correspond to any of the recognized species of GPAC genera. In this article, we report the phenotypic characteristics of the strains and the results of a phylogenetic analysis and, based on the results presented, a new genus and species are described.

Strains WAL 2038E and WAL $1855 \mathrm{C}^{\mathrm{T}}$ and the reference strains Parvimonas micra ATCC $33270^{\mathrm{T}}$, Finegoldia magna CCUG $17636^{\mathrm{T}}$, Helcococcus sueciensis CCUG $47334^{\mathrm{T}}$ and Helcococcus kunzii CCUG $32213^{\mathrm{T}}$ were included in the present study. Clinical specimens were obtained at Olive View-UCLA Hospital. The specimens were collected, transported and processed as outlined by JousimiesSomer et al. (2002). Anaerobic conditions consisted of a gas mixture of $5 \% \mathrm{CO}_{2}, 5 \% \mathrm{H}_{2}$ and $90 \% \mathrm{~N}_{2}$. Microaerophilic conditions were obtained using an automated evacuation-replacement system [Anoxomat (Mart BV Laboratorium); microaerophilic cycle]. All testing was done on 24-72 h pure cultures on plates of Brucella blood agar (Anaerobe Systems), CDC blood agar (Becton 
Dickinson Microbiology) or trypticase soy blood agar (TSBA; Becton Dickinson Microbiology). Single colonies were selected, described and subcultured to assure purity and to determine whether the organism was aerobic, anaerobic or microaerophilic, and were confirmed as anaerobes if there was no growth in aerobic or microaerophilic environments.

The strains were characterized biochemically by using a combination of conventional tests and commercially available biochemical kits. Conventional identification of bacteria was performed based on Gram stain, colony morphology, susceptibility to special-potency antibiotic discs [colistin, vancomycin, kanamycin and sodium polyanethol sulfonate (SPS)] and other biochemical reactions according to Jousimies-Somer et al. (2002). The commercial biochemical kits Rapid ID 32A and API ZYM (bioMérieux) were used according to the manufacturer's instructions and the results were graded using a colour chart supplied by the manufacturer. All biochemical tests were performed in duplicate. Carbohydrate fermentation tests were performed using pre-reduced, anaerobically sterilized peptone-yeast (PY) broth tubes (Anaerobe Systems) with the addition of different carbohydrates. The strains were grown in PY and peptone-yeast-glucose (PYG) broth (Anaerobe Systems) for analysis of metabolic end products (short-chain volatile and non-volatile fatty acids) by GLC.

Genomic DNA was extracted and purified from bacterial cells in the mid-exponential growth phase by using a QIAamp DNA Mini kit (Qiagen). 16S rRNA gene fragments were amplified by standard methods (Song et al., 2003). Two subregions of the 16S rRNA gene were amplified by using two pairs of primers. The first part of the $16 \mathrm{~S}$ rRNA gene was defined as an approximately 800 bp region between primers 8UA (5'-AGAGTTTGATCCTGGCTCAG-3') and 907B (5'-CCGTCAATTCMTTTAGTTT- $3^{\prime}$ ). The second part, defined as an approximately $700 \mathrm{bp}$ sequence between primers 774A (5'-GTAGTCCACGCTGTAAACGATG- ${ }^{\prime}$ ) and 1485B (5'-TACGGTTACCTTGTTACGAC-3'), was sequenced to obtain the complete 16S rRNA gene sequence. PCR was performed for 35 cycles of $30 \mathrm{~s}$ at $95{ }^{\circ} \mathrm{C}, 30 \mathrm{~s}$ at $45{ }^{\circ} \mathrm{C}$ and $1 \mathrm{~min}$ at $72{ }^{\circ} \mathrm{C}$, with a final extension at $72{ }^{\circ} \mathrm{C}$ for $5 \mathrm{~min}$. The PCR products were excised from a $1 \%$ agarose gel after electrophoresis and purified using a QIAquick Gel Extraction kit (Qiagen). Purified PCR products were sequenced directly with BigDye Terminator cycle sequencing kits (Applied Biosystems) on an ABI 3100 Avant Genetic System (Applied Biosystems). The sequences were analysed by comparison of the consensus sequences with GenBank sequences by using Ribosomal Database Project (RDP-II) and BLAST (Benson et al., 1997) software, and the percentage similarity to other sequences was determined. Closely related sequences were retrieved from GenBank and were aligned with the newly determined sequences by using the program CLUSTAL $\mathrm{W}$ (Thompson et al., 1994). Phylogenetic and molecular evolutionary analyses were constructed by using the neighbour-joining algorithm within MEGA version 4 (Tamura et al., 2007).

Strain WAL $1855 C^{\mathrm{T}}$ was isolated from a sacral pilonidal cyst aspirate from an immunocompetent patient and strain WAL 2038E was obtained from an abdominal wall abscess of a morbidly obese diabetic patient at an insulin injection site; no other clinical information was given. Strain WAL $1855 \mathrm{C}^{\mathrm{T}}$ was isolated together with strains of Solobacterium moorei, Streptococcus anginosus/Streptococcus constellatus, Parvimonas micra and Bilophila wadsworthia and strains showing $98 \% 16 \mathrm{~S}$ rRNA gene sequence similarity to an uncultured member of the Clostridiales, and WAL 2038E was isolated with strains of Corynebacterium simulans, Actinomyces europaeus, Mobiluncus curtisii, Peptoniphilus asaccharolyticus, Actinobaculum schaalii and Anaerococcus vaginalis. The majority of these isolates were present in counts of $\geqslant 10^{5}$ c.f.u. $\mathrm{ml}^{-1}$ (WAL $1855 \mathrm{C}^{\mathrm{T}}, 2 \times 10^{5}$ c.f.u. $\mathrm{ml}^{-1}$; WAL $2038 \mathrm{E}, 1 \times 10^{5}$ c.f.u. $\mathrm{ml}^{-1}$ ). Comparative $16 \mathrm{~S}$ rRNA gene sequencing studies on the isolates demonstrated that the novel strains did not correspond to any of the recognized species in the GenBank database. The novel species were found to be Gram-stain-positive, coccoid organisms. Cells were 0.5-0.6 $\mu \mathrm{m}$ in diameter and occurred in pairs and short chains. Colonies on Brucella blood agar plates at 5 days were grey, flat or low-convex, circular, entire, white and opaque with a diameter of $2-3 \mathrm{~mm}$. They grew well anaerobically, but no growth occurred following subculture in air or in atmospheres of 2 or $6 \% \mathrm{O}_{2}$. The novel strains were sensitive to special-potency identification discs containing kanamycin $(1000 \mu \mathrm{g})$ and vancomycin $(5 \mu \mathrm{g})$ and resistant to discs containing colistin sulfate $(10 \mu \mathrm{g})$ and SPS $(1000 \mu \mathrm{g})$.

The novel strains were negative for catalase, urease and reduction of nitrate and produced indole. The unknown strains and Parvimonas micra ATCC $33270^{\mathrm{T}}$ and F. magna CCUG $17636^{\mathrm{T}}$ were asaccharolytic; they did not produce acid from glucose or other carbohydrates. The two tested Helcococcus strains, $H$. kunzii CCUG $32213^{\mathrm{T}}$ and $H$. sueciensis CCUG $47334^{\mathrm{T}}$, produced acid from lactose and trehalose. Based on results from the literature (Collins et al., 1999), Helcococcus ovis produced acid from glucose but not from lactose or trehalose. In PYG broth, major amounts of lactic and oxaloacetic acids and moderate amounts of acetic, butyric and succinic acids were produced by the novel isolates. Parvimonas micra ATCC $33270^{\mathrm{T}}$ and F. magna CCUG $17636^{\mathrm{T}}$ produced major amounts of acetic acid and small amounts of lactic and succinic acids.

Using the Rapid ID 32A kit, the two novel isolates produced identical profiles. Positive reactions were obtained for arginine dihydrolase, arginine arylamidase, proline arylamidase, leucyl glycine arylamidase, phenylalanine arylamidase (weak), leucine arylamidase, tyrosine arylamidase (weak), alanine arylamidase, glycine arylamidase, histidine arylamidase and serine arylamidase. All the other tests were negative. Neither strain hydrolysed 
aesculin or gelatin. According to the API ZYM system, the unknown strains were positive for leucine arylamidase and esterase (C4) only. Distinguishing results from the Rapid ID 32A kit and other biochemical tests between the novel strains and members of the phylogenetically most closely related genera are given in Table 1.

To assess the genotypic affinity between the unknown bacteria and their relationship with other taxa, their $16 \mathrm{~S}$ rRNA gene sequences were determined. The sequences of the novel strains were identical (100\% sequence similarity) and were most closely related to that of bacterium N14-24 (approx. 99\% sequence similarity) in GenBank. Bacterium N14-24 was isolated by Hooper et al. (2006) from oral squamous cell carcinoma tissue, but the properties of the strain have not been described. Investigation of the most closely related described species revealed that the novel strains were members of the phylum Firmicutes and represent a previously unknown lineage within Clostridium cluster XIII, exhibiting $87 \%$ sequence similarity with Parvimonas micra ATCC $33270^{\mathrm{T}}$ and $86 \%$ sequence similarity with strains of F. magna, H. sueciensis, $H$. kunzii and H. ovis. The novel strains could be distinguished from Parvimonas micra and F. magna by the presence of indole production and the absence of pyroglutamic acid arylamidase activity. Further, the obligately anaerobic novel isolates could be differentiated from facultatively anaerobic Helcococcus species according to atmospheric growth requirements (Collins et al., 1993, 1999,

Table 1. Phenotypic characteristics of strain WAL $1855 C^{\top}$ and the type strains of the phylogenetically most closely related species

Strains: 1, Murdochiella asaccharolytica gen. nov., sp. nov. WAL $1855 \mathrm{C}^{\mathrm{T}}$; 2, Parvimonas micra CCUG $46357^{\mathrm{T}}$; 3, F. magna CCUG $17636^{\mathrm{T}}$; 4, H. kunzii CCUG $32213^{\mathrm{T}}$; 5, H. sueciensis CCUG $47334^{\mathrm{T}}$; 6 , H. ovis CCUG $37441^{\mathrm{T}}$. Data for reference strains were obtained from Collins et al. (1999). +, Positive; -, negative; v, variable; ND, no data available.

\begin{tabular}{|c|c|c|c|c|c|c|}
\hline Characteristic & 1 & 2 & 3 & 4 & 5 & 6 \\
\hline \multicolumn{7}{|l|}{ Acid production from: } \\
\hline Lactose & - & - & - & + & + & - \\
\hline Trehalose & - & - & - & + & + & - \\
\hline Glucose & - & - & - & + & + & + \\
\hline \multicolumn{7}{|l|}{ Production of: } \\
\hline Alkaline phosphatase & - & + & $\mathrm{v}$ & - & + & + \\
\hline$\beta$-Glucosidase & - & - & - & + & - & - \\
\hline Indole & + & - & - & - & - & $\mathrm{ND}$ \\
\hline Pyroglutamic acid arylamidase & - & + & + & + & - & - \\
\hline$N$-Acetyl- $\beta$-glucosaminidase & - & - & - & + & + & - \\
\hline Metabolic end products ${ }^{\star}$ & $\begin{array}{r}\mathrm{L}, \mathrm{a}, \\
\mathrm{b}, \mathrm{s}\end{array}$ & A, $1, s$ & A, $1, s$ & $\mathrm{a}, \mathrm{s}$ & $\mathrm{a}$ & $\mathrm{ND}$ \\
\hline
\end{tabular}

${ }^{*} \mathrm{~A} / \mathrm{a}$, Acetic acid; b, butyric acid; L/l, lactic acid; s, succinic acid. Capitals indicate major amounts produced; lower-case letters indicate small amounts produced.
2004); Helcococcus species produce heavy growth of pinpoint colonies on $5 \%$ sheep blood agar and chocolate agar plates under a $\mathrm{CO}_{2}$-enriched (5\%) atmosphere, whereas the novel strains only grow under anaerobic conditions. A tree depicting the phylogenetic affinity of strains WAL $1855 \mathrm{C}^{\mathrm{T}}$ and WAL 2038E is shown in Fig. 1 and confirmed the placement of the unknown bacterium in Clostridium cluster XIII. On the basis of low level of $16 \mathrm{~S}$ rRNA gene sequence similarity $(<87 \%)$, it is evident from the branching pattern in the tree that the strains constitute a novel lineage within Clostridium cluster XIII.

Based on the previously mentioned characteristics, the isolates showed some resemblance to members of a number of genera that encompass species formerly assigned to the genus Peptostreptococcus (e.g. Parvimonas, Finegoldia, Peptoniphilus, Gallicola and Anaerococcus) within Clostridium cluster XIII (Collins et al., 1994; Ezaki et al., 2001). However, the genotypic and phenotypic test results clearly demonstrated that the unknown cocci were distinct from the phylogenetically closest members of GPAC genera (Fig. 1; Table 1). Therefore, we propose that the unidentified coccus-shaped bacterium be assigned to a novel genus and species, Murdochiella asaccharolytica gen. nov., sp. nov.

\section{Description of Murdochiella gen. nov.}

Murdochiella (Mur.do.chi.el'la. N.L. fem. dim. n. Murdochiella named to honour Dr David A. Murdoch, British microbiologist, who has contributed so much to our knowledge of anaerobic bacteriology).

Cells are cocci, Gram-stain-positive and non-motile. Obligately anaerobic. Indole-positive. Catalase- and ureasenegative. Nitrate is not reduced. Carbohydrates are not fermented. In PY and PYG broth, major amounts of lactic acid and moderate amounts of acetic, butyric and succinic acids are produced. Bile-sensitive. Do not hydrolyse aesculin or gelatin. The type species is Murdochiella asaccharolytica.

\section{Description of Murdochiella asaccharolytica sp. nov.}

Murdochiella asaccharolytica (a.sac.cha.ro.ly'ti.ca. Gr. pref. a- not; Gr. n. saccharon sugar; N.L. fem. adj. lytica from Gr. adj. lutikos able to lyse, able to loose; N.L. fem. adj. asaccharolytica not digesting sugar).

Displays the following properties in addition to those described for the genus. Cells are $0.5-0.6 \mu \mathrm{m}$ in diameter and occur in pairs and short chains. Colonies on Brucella blood agar plates at 5 days are grey, flat or low-convex, circular, entire, white and opaque with a diameter of $2-$ $3 \mathrm{~mm}$. Asaccharolytic; acid is not produced from glucose or other sugars. Using the Rapid ID 32A kit, positive reactions are obtained for arginine dihydrolase, arginine arylamidase, phenylalanine arylamidase (weak), proline arylamidase, leucine arylamidase, leucyl glycine arylamidase, alanine arylamidase, tyrosine arylamidase (weak), histidine arylamidase and serine arylamidase. According to 


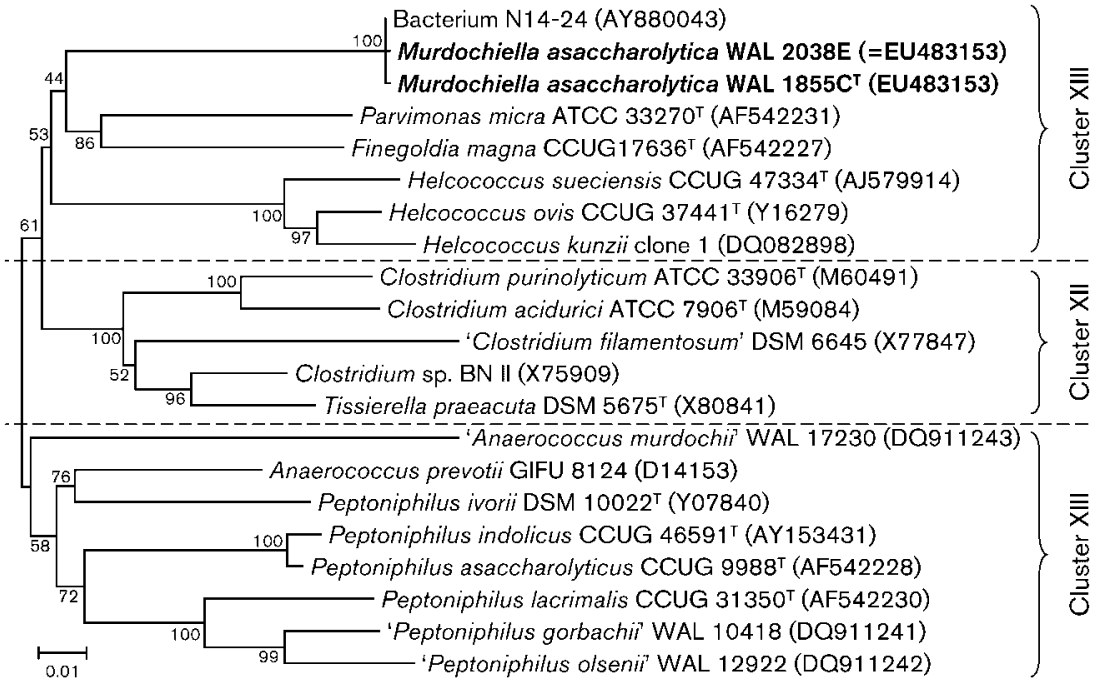

Fig. 1. Phylogenetic position of strains WAL $1855 C^{\top}$ and WAL 2038E within the Clostridiales. The alignment was performed with 16S rRNA gene sequences of the most closely related species of Clostridium clusters XII and XIII. Accession numbers are indicated in parentheses. Unrooted tree constructed using the neighbour-joining algorithm established by MEGA version 4 (Tamura et al., 2007). Bootstrap values are displayed as percentages on the relevant branches. Bar, 0.01 inferred substitutions per nucleotide position. the API ZYM system, leucine arylamidase and esterase (C4) are positive. All the other tests are negative; aesculin and gelatin are not hydrolysed. Sensitive to vancomycin $(5 \mu \mathrm{g})$ and kanamycin $(1000 \mu \mathrm{g})$, resistant to colistin sulfate $(10 \mu \mathrm{g})$ and SPS $(1000 \mu \mathrm{g})$ identification discs. Strains have been isolated from human clinical specimens.

The type strain is WAL $1855 \mathrm{C}^{\mathrm{T}}$ (=ATCC BAA-1631 ${ }^{\mathrm{T}}$ $=$ CCUG $55976^{\mathrm{T}}$ ). Strain WAL $2038 \mathrm{E}$ is a second strain of the species.

\section{Acknowledgements}

This work has been supported by DOD grant no. W81XWH-0510134 and by VA Merit Review funds.

\section{References}

Benson, D. A., Boguski, M. S., Lipman, D. J. \& Ostell, J. (1997). GenBank. Nucleic Acids Res 25, 1-6.

Collins, M. D., Facklam, R. R., Rodrigues, U. M. \& Ruoff, K. L. (1993). Phylogenetic analysis of some Aerococcus-like organisms from clinical sources: description of Helcococcus kunzii gen. nov., sp. nov. Int J Syst Bacteriol 43, 425-429.

Collins, M. D., Lawson, P. A., Willems, A., Cordoba, J. J., FernandezGarayzabal, J., Garcia, P., Cai, J., Hippe, H. \& Farrow, J. A. E. (1994). The phylogeny of the genus Clostridium: proposal of five new genera and eleven new species combinations. Int J Syst Bacteriol 44, 812-826.

Collins, M. D., Falsen, E., Foster, G., Monasterio, L. R., Dominguez, L. \& Fernandez-Garazabal, J. F. (1999). Helcococcus ovis sp. nov., a Gram-positive organism from sheep. Int J Syst Bacteriol 49, 14291432.
Collins, M. D., Falsen, E., Brownlee, K. \& Lawson, P. A. (2004). Helcococcus sueciensis sp. nov., isolated from a human wound. Int $J$ Syst Evol Microbiol 54, 1557-1560.

Downes, J. \& Wade, W. G. (2006). Peptostreptococcus stomatis sp. nov., isolated from the human oral cavity. Int J Syst Evol Microbiol 56, 751754.

Ezaki, T., Kawamura, Y., Li, N., Li, Z. Y., Zhao, L. \& Shu, S. (2001). Proposal of the genera Anaerococcus gen. nov., Peptoniphilus gen. nov. and Gallicola gen. nov. for members of the genus Peptostreptococcus. Int J Syst Evol Microbiol 51, 1521-1528.

Finegold, S. M. (1977). Anaerobic Bacteria in Human Disease. New York: Academic Press.

Finegold, S. M., Song, Y. \& Liu, C. (2002). Taxonomy - general comments and update on taxonomy of Clostridia and anaerobic cocci. Anaerobe 8, 283-285.

Hooper, S. J., Crean, S. J., Lewis, M. A., Spratt, D. A., Wade, W. G. \& Wilson, M. J. (2006). Viable bacteria present within oral squamous cell carcinoma tissue. J Clin Microbiol 44, 1719-1725.

Jousimies-Somer, H., Summanen, P., Citron, D. M., Baron, E. J., Wexler, H. M. \& Finegold, S. M. (2002). Wadsworth-KTL Anaerobic Bacteriology Manual, 6th edn. Belmont, CA: Star Publishing.

Murdoch, D. A. (1998). Gram-positive anaerobic cocci. Clin Microbiol Rev 11, 81-120.

Song, Y., Liu, C., Mc Teague, M. \& Finegold, S. M. (2003). 16S ribosomal DNA sequence-based analysis of clinically significant Gram-positive anaerobic cocci. J Clin Microbiol 41, 1363-1369.

Tamura, K., Dudley, J., Nei, M. \& Kumar, S. (2007). MEGA4: molecular evolutionary genetics analysis (MEGA) software version 4.0. Mol Biol Evol 24, 1596-1599.

Thompson, J. D., Higgins, D. G. \& Gibson, T. J. (1994). ClusTAL W: improving the sensitivity of progressive multiple sequence alignment through sequence weighting, position-specific gap penalties and weight matrix choice. Nucleic Acids Res 22, 4673-4680. 